\title{
Stability of plane wave solutions in complex Ginzburg-Landau equation with delayed feedback*
}

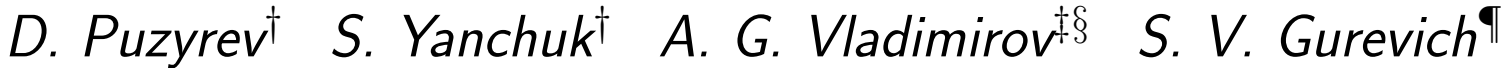

\begin{abstract}
We perform bifurcation analysis of plane wave solutions in one-dimensional complex cubicquintic Ginzburg-Landau equation with delayed feedback. Our study reveals how multistability and snaking behavior of plane waves emerge as time delay is introduced. For intermediate values of the delay, bifurcation diagrams are obtained by a combination of analytical and numerical methods. For large delays, using an asymptotic approach we classify plane wave solutions into strongly unstable, weakly unstable, and stable. The results of analytical bifurcation analysis are in agreement with those obtained by direct numerical integration of the model equation.
\end{abstract}

Keywords: delay differential equation, stability theory, large delay, periodic solutions.

\section{Introduction}

The complex Ginzburg-Landau equation (CGLE) plays an important role in modeling of various natural phenomena including nonlinear optical waves, second-order phase transitions, Rayleigh-Bénard convection, and superconductivity [5, 3]. It is an amplitude equation describing the onset of instability near an Andronov-Hopf bifurcation in spatially extended dynamical systems [19]. In nonlinear optics, equations of CGLE type are widely used to describe such phenomena as mode-locking in lasers [26, 2, 9], short pulse propagation in optical transmission lines [15], dynamics of multimode lasers, and transverse pattern formation in nonlinear optical media [31, 28]. While classical cubic CGLE describes a supercritical bifurcation, in the case of subcritical instability this equation is augmented with the fifth-order terms to allow the existence of stable pulselike solutions [29, 12 .

*We acknowledge the German Research Foundation (DFG) for financial support in the framework of the Collaborative Research Center SFB 910, and Erasmus Mundus TRIPLEI2011332 mobility program grant. A. G. Vladimirov acknowledges the support from the SFI E.T.S. Walton award and FP7 ITN PROPHET, grant 264687.

$\dagger$ Institute of Mathematics, Humboldt University of Berlin, Unter den Linden 6, D-10099 Berlin, Germany (puzyrev@math.hu-berlin.de).

$\ddagger$ Weierstrass Institute for Applied Analysis and Stochastics, Mohrenstrasse 39, D-10117 Berlin, Germany

$\S$ Cork Institute of Technology, Rossa Ave, Bishopstown, Cork, Ireland

ฯ Institute for Theoretical Physics, University of Münster, Wilhelm-Klemm-Str. 9, D-48149 Münster, Germany 
Here we focus on one-dimensional delayed cubic-quintic CGLE for the complex amplitude $A(x, t)$

$$
\partial_{t} A=\left(\beta+\frac{i}{2}\right) \partial_{x x} A+\delta A+(\epsilon+i)|A|^{2} A+(\mu+i \nu)|A|^{4} A+\eta e^{i \varphi} A(x, t-\tau) .
$$

The parameter $\beta>0$ is the diffusion coefficient, second-order dispersion (diffraction) is scaled to $1 / 2$, and $\delta$ describes the linear loss or gain. Parameters $\epsilon, \mu$, and $\nu$ determine the shape of the nonlinearity. In particular, there are two qualitatively different cases: $\epsilon>0$ and $\epsilon<0$ corresponding to destabilizing and stabilizing role of the cubic nonlinearity. Equivalently, this leads to either subcritical or supercritical destabilization mechanisms for the homogeneous steady state $A=0$. In this work, we take the values $\varepsilon<0$, $\mu=\nu=0$ for the supercritical case (cubic CGLE), and $\varepsilon>0, \mu<0$ for the subcritical case. Parameters $\eta$ and $\varphi$ determine the feedback rate and phase, respectively, whereas $\tau$ is the delay time. Model equation (1) can describe, for instance, a broad area optical system with delayed optical feedback [30, 10]. Notice that in the absence of delayed feedback term, $\eta=0$, Eq. (1) becomes the classical cubic-quintic CGLE [5, 23].

Although CGLE possesses a variety of different solutions [1, 27, 3, 16], in this work we focus on the simplest plane waves of the form $A=a_{0} e^{i q x+i \omega t}$. The stability of the trivial homogeneous solution $A=0$ is studied as well. Criteria of the stability of plane wave solutions in the quintic CGLE without delay were briefly described in [23]. The stabilization of plane waves in one-dimensional and two-dimensional cubic CGLE by a combination of spatially shifted and temporally delayed non-invasive feedback was investigated in [17, 20] for the case when delay time and space shift are in the resonance with plane wave spatial and temporal wavenumbers. In this paper, we study cubic-quintic CGLE (as well as cubic CGLE as a special case) with arbitrary delay time and phase of the feedback, including the long delay limit case. For small delay times, there appears a single plane wave for every allowed spatial wavenumber $q$. The local stability of such solutions can be studied by calculating a dispersion relation for a given plane wave solution [23]. As time delay becomes comparable or longer than the characteristic time scale in the system the number of plane waves for each admissible wavenumber grows linearly with $\tau$. Moreover, the stability of each plane wave is no longer determined by a single classical dispersion relation, but a set of dispersion relations, which correspond to various "delay-induced modes". This set of dispersion relations is conveniently described using the methods from [32, 34, 14, 24, 8] by adding an additional dimension to the dispersion relation and studying a so called "hybrid dispersion relation", which is a function of two arguments. Performing such a stability analysis, we identify a large set of emerging asymptotically stable plane waves. Another contribution of this work is that we present a way how one can conveniently describe and visualize the whole set of plane waves and their stability in system (1). As an interesting observation, we find that the branches of plane waves exhibit a snaking behavior as the linear gain parameter $\delta$ is changed.

This paper is organized as follows: in Section 2 we start with the stability analysis of the homogeneous solution $A=0$. In addition, in this section we introduce some important ideas that will be used in a technically more elaborated way in Section 3, where the existence and stability of the plane wave solutions $A=a_{0} e^{i q x+i \omega t}$ is studied. Finally, the conclusions are given in Section 4. 


\section{Stability analysis of the homogeneous solution}

\subsection{The case without delayed feedback}

In this section we start with the stability analysis of the trivial homogeneous steady state solution $A(x, t)=0$. Let us briefly recall the case when the feedback is absent, i.e. $\eta=0$ [23, 17]. By substituting the perturbations of the form $e^{i q x+\lambda t}$ in the linearized equation, we obtain the characteristic equation

$$
\chi(\lambda)=\lambda-\delta+\left(\beta+\frac{i}{2}\right) q^{2}=0 .
$$

Here $q$ is a spatial wavenumber of the perturbation and $\lambda$ determines the growth rate. The corresponding dispersion relation reads

$$
\lambda(q)=\delta-\left(\beta+\frac{i}{2}\right) q^{2}
$$

When all the eigenvalues $\lambda(q)$ have negative real parts, the homogeneous solution is asymptotically stable. Since $\beta$ is positive, we conclude that the trivial solution is unstable for $\delta>0$ and stable if $\delta<0$. The most unstable wavenumber is $q=0$.

\subsection{Case of delay $\tau$}

For nonzero feedback rate the characteristic equation for the homogeneous solution becomes

$$
\chi_{1}(\lambda)=\lambda-\delta-\eta e^{i \varphi} e^{-\lambda \tau}+\left(\beta+\frac{i}{2}\right) q^{2}=0 .
$$

The Andronov-Hopf bifurcation curves in the parameter plane $(\eta, \delta)$ can be found in a parametric form. To this end we substitute $\lambda=i \omega$ into characteristic equation (3)

$$
i \omega-\delta-\eta e^{i \varphi} e^{-i \omega \tau}+\left(\beta+\frac{i}{2}\right) q^{2}=0
$$

By separating real and imaginary parts of equation (4), we obtain two relations

$$
\eta(\omega)=\frac{\frac{q^{2}}{2}+\omega}{\sin (\varphi-\omega \tau)}, \quad \delta(\omega)=\beta q^{2}-\frac{\cos (\varphi-\omega \tau)\left(\frac{q^{2}}{2}+\omega\right)}{\sin (\varphi-\omega \tau)}
$$

defining the Andronov-Hopf bifurcation curves with the imaginary part of the critical eigenvalue $\omega$ being a free parameter. Figure 1 shows these bifurcation curves in the plane of two parameters, linear gain $\delta$ and feedback rate $\eta$. The stability region of the trivial solution where real parts of all the eigenvalues $\lambda(q)$ are negative is shown in gray. A destabilization with respect to a given wavenumber $q$ takes place when crossing the boundary of this region from inside. Figures $1(\mathrm{a}, \mathrm{b})$ present the Andronov-Hopf bifurcation curves for the wavevector $q=0$ at different values of the feedback phase, see also [21, 17]. For non-zero values of $q$, the instability region shifts to higher values of $\delta$, as shown in Fig. 1(c). Therefore, the destabilization of the trivial homogeneous steady state first occurs at the most unstable wavenumber $q=0$. For larger delay times the set of bifurcation curves becomes more dense, as it is seen from Fig. 1(d). 

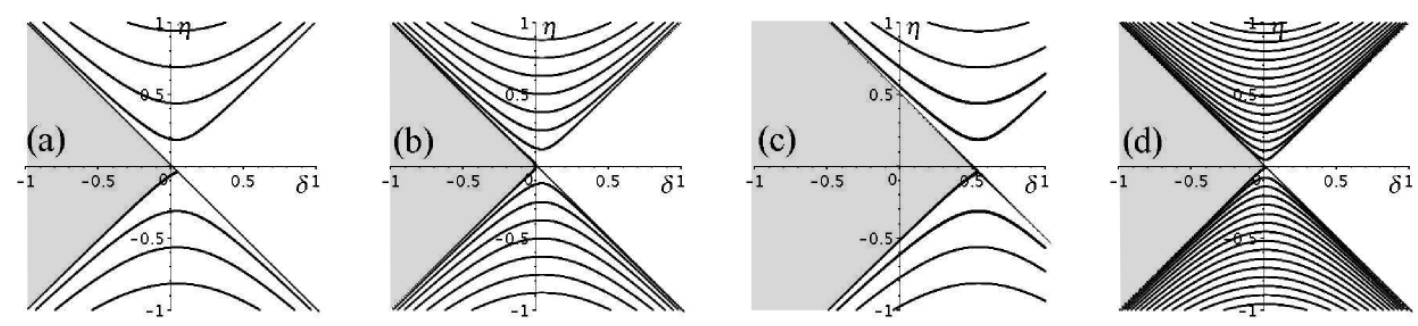

Fig. 1: Andronov-Hopf bifurcation curves for the trivial homogeneous solution $A=0$ of Eq. (1) in $(\delta, \eta)$ plane. The curves are given in a parametric form (5). Stability region of the solution $A=0$ where the real part of the eigenvalue corresponding to the most unstable mode $q=0$ is negative is shown in gray. Parameter values: (a) $\tau=25, q=0, \varphi=0$, (b) $\tau=25, q=0, \varphi=\pi / 2$, (c) $\tau=25, q=1, \varphi=0$, (d) $\tau=100, q=0, \varphi=0$. In all figures $\beta=0.5$.

\subsection{The case of large delay}

Delay time $\tau$ for optical systems can exceed the internal time scales of the system by several orders of magnitude [25, 6, 13, 7]. To study this case, we use the asymptotic technique similar to that used in [32, 34, 14, 24]. According to these results, there are two types of delay-induced instabilities: strong instability and weak instability. Strong instability appears when there exists an eigenvalue (or Lyapunov exponent, for the periodic or chaotic case), which tends to some constant value $\lambda(\tau) \rightarrow \lambda_{0}$ with $\Re\left[\lambda_{0}\right]>0$ as time delay increases. In this case, the contribution of the term $e^{-\lambda \tau}$ in the characteristic equation (3) can be neglected and we arrive to the following condition for the strong instability of the mode with the wavenumber $q$

$$
\lambda_{0}=\delta+\left(\beta+\frac{i}{2}\right) q^{2}>0
$$

This instability condition coincides, in fact, with that for the feedback-free case. The most unstable mode $q=0$ gives the condition for the strong instability of the solution $A=0$ :

$$
\delta>0 .
$$

Another type of instability, the weak one, can be described by a pseudo-continuous spectrum of eigenvalues, which scales as

$$
\lambda=\frac{\gamma(\xi)}{\tau}+i \xi
$$

in the limit $\tau \rightarrow \infty$. More specifically, it was proved in [14, 24] that this spectrum is converging to a set of continuous curves (7) parametrized by $\xi$. Moreover, the leading terms of the real parts $\gamma(\xi)$ can be found explicitly by substituting (7) into the characteristic equation (3) and neglecting small terms of order $1 / \tau$. In our case, we obtain the equation

$$
i \xi-\delta-\eta e^{i \varphi} e^{-\gamma} e^{-i \xi \tau}+\left(\beta+\frac{i}{2}\right) q^{2}=0
$$

which is solved explicitly with respect to $\gamma$ :

$$
\gamma(\xi, q)=-\frac{1}{2} \ln \frac{\left(\delta-\beta q^{2}\right)^{2}+\left(\xi+\frac{1}{2} q^{2}\right)^{2}}{\eta^{2}} .
$$


In Eq. (9), we write an additional argument $q$, which indicates the dependence of the real part (rescaled by $1 / \tau$ ) of the eigenvalues on the wavenumber. The function $\gamma$ of two arguments extends naturally the dispersion relation, which is used [4] for partial differential equations without delayed feedback. Indeed, for a fixed $\xi$, the relation (9) determines the stability of the homogeneous state with respect to the perturbations with the wavenumber $q$, i.e. it is the dispersion relation. The new variable $\xi$ stands for the delay induced modes, which appear additionally due to the delay. The homogeneous solution is locally asymptotically stable when $\gamma(\xi, q)<0$.

Figure 2 shows the surfaces of $\gamma(\xi, q)$ calculated for different parameters. The red curve shows the level line $\gamma=0$ given by the relation

$$
\left(\delta-\beta q^{2}\right)^{2}+\left(\frac{q^{2}}{2}+\xi\right)^{2}=\eta^{2} .
$$

Equation (10) defines an ellipse in $\xi$ and $q^{2}$ coordinates. The trivial solution $A=0$ is unstable if at least part of the ellipse lies in the half-plane $q^{2}>0$. Simple calculations show that, for $\beta>0$, this is equivalent to the condition

$$
\delta>-|\eta|
$$

Hence, the inequality (11) gives the weak instability condition for the solution $A=0$, see Fig. 3. The corresponding critical wave numbers $\xi_{c}$ and $q_{c}$ correspond to the maximum of the quantity $\gamma(\xi, q)$. For $\delta \leq 0$, we have $q_{c}=0$ and $\xi_{c}=0$. Under the condition

$$
\delta>|\eta|
$$

there are two separated regions of unstable wavenumbers $\xi$ and $q$, for which $\gamma(\xi, q)>0$, see Fig. 2(a). These regions are symmetric with respect to the $\xi$-axis, $q=0$. Otherwise, when only first of the two inequalities, (11) and (12), is satisfied, there is a single symmetric region of unstable wavenumbers, see Fig. 2(b). The boundary defined by the inequality (12) is shown in Fig. 3 by a dashed line. Figure2(c) illustrates the case when the eigenvalue spectrum is located in the left half-plane of the complex plane, $\gamma(\xi, q)<0$, and the homogeneous state $A=0$ is stable.

The complete bifurcation diagram for the homogeneous state in the case of long delay is summarized in Fig. 3, where the regions of strong and weak instability are shown. It is instructive to compare this bifurcation diagram obtained in the limit $\tau \rightarrow 0$ with the exact bifurcation curves for different values of $\tau$ shown at Figs. 11(a,b,d).

\section{Plane wave solutions}

In this section we study existence and stability of plane wave solutions $A=a_{0} e^{i q x+i \omega t}$ of Eq. (1). As for the homogeneous solution, we start with the case without delayed feedback in Sec. 3.1, and then consider the case with finite delay in Sec. 3.2, Further, in Sec. 3.3 we study analytically the limit of a large delay time $\tau$, which allows for a deeper insight into the stability properties of plane wave solutions as compared to the arbitrary $\tau$ case. Finally, in Sec. 3.4 the results of numerical simulations are presented. 

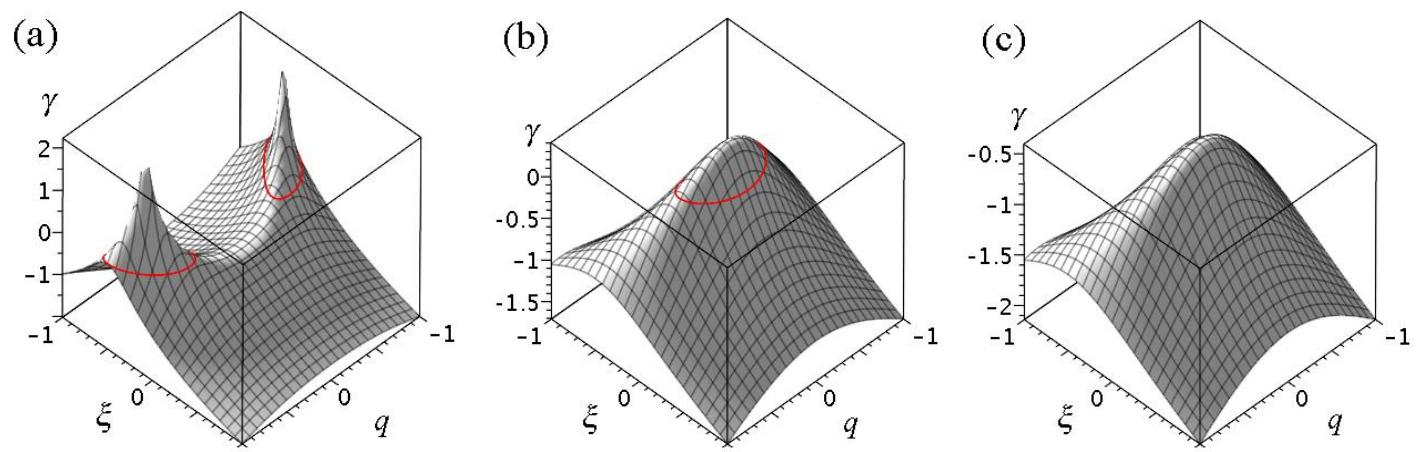

Fig. 2: Dispersion relation $\gamma(\xi, q)$, given by Eq. (9) for the homogeneous state $A=0$ at large $\tau$. Here $\xi$ and $q$ stand for the delay induced and the spatial wavenumber of the perturbations. If $\gamma<0$ for all $\xi$ and $q$ and no strong instability occurs, the homogeneous state is stable. Parameter values: (a) unstable case with two regions of unstable wavenumbers: $\delta=0.3, \eta=0.2$, (b) unstable case with one unstable region: $\delta=-0.2, \eta=0.3$, (c) stable case: $\delta=-0.3, \eta=0.2$. Red curve shows the level lines $\gamma=0$. In all figures $\beta=0.5$.

\subsection{The case without delayed feedback}

Substituting $A=a_{0} e^{i q x+i \omega t}$ into Eq. (1), we obtain the relation between the unknown amplitude $a_{0}$, wavenumber $q$, and frequency $\omega$ of the plane wave solution:

$$
i \omega=-\left(\beta+\frac{i}{2}\right) q^{2}+\delta+(\epsilon+i) a_{0}^{2}+(\mu+i \nu) a_{0}^{4}
$$

Due to the symmetry property of the CGLE $A \rightarrow-A$, this equation is symmetric under the reflection $a_{0} \rightarrow-a_{0}$. Hence, we restrict our analysis to the case $a_{0} \geq 0$. The real and imaginary parts of Eq. 13) give the expressions for the amplitude $a_{0}^{2}(q)$ and the frequency $\omega(q)$ at a given wavenumber $q$ :

$$
a_{0}^{2}(q)=\frac{-\epsilon \pm \sqrt{\epsilon^{2}-4 \mu\left(\delta-\beta q^{2}\right)}}{2 \mu}, \quad \omega(q)=-\frac{q^{2}}{2}+a_{0}^{2}(q)+\nu a_{0}^{4}(q) .
$$

In particular, for the cubic CGLE (supercritical case with $\mu=\nu=0$ ) we obtain

$$
a_{0}^{2}(q)=\sqrt{\frac{\beta q^{2}-\delta}{\epsilon}}, \quad \omega(q)=-\frac{q^{2}}{2}+\frac{\beta q^{2}-\delta}{\epsilon} .
$$

Figures 4 (a) and (b) show the amplitude of the plane wave $a_{0}(\delta)$ as a function of the gain parameter $\delta$ for the supercritical and subcritical case, respectively. The branch of plane wave solutions with a given $q$ emerges from the homogeneous state via Hopf bifurcation at $\delta=\beta q^{2}$. For the parameter values of Fig. 4(b) corresponding to the quintic CGLE, it bifurcates subcritically from $A=0$ and undergoes a fold bifurcation at $\delta=\frac{\epsilon^{2}}{4 \mu}+\beta q^{2}$.

Note that plane wave solutions of the CGLE without delay in supercritical case show the classical Benjamin-Feir scenario [23, 3]. Let us shortly discuss the stability of plane waves in the subcritical case of the quintic CGLE. Although the main ideas of this analysis are known from e.g. [23], there are still some details, which are not explained in [23], but are important for our further analysis. The perturbed plane wave solutions have the form

$$
A(x, t)=\left(a_{0}+a_{p}\right) e^{i q x+i \omega t},
$$




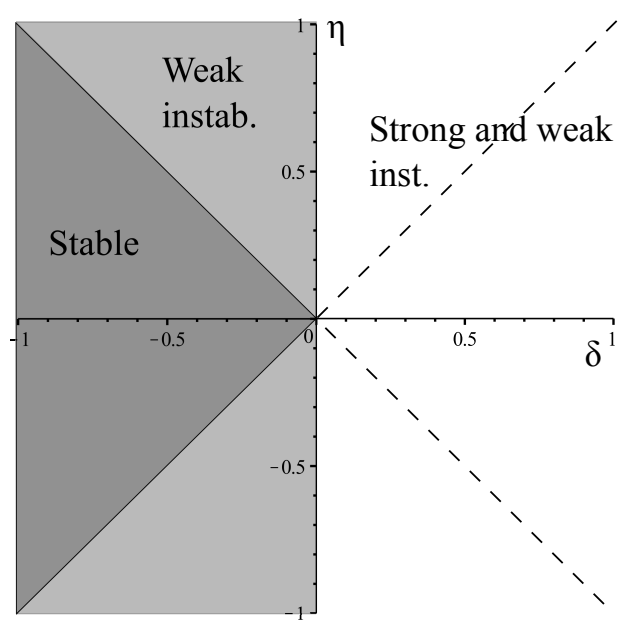

Fig. 3: Bifurcation diagram of the homogeneous solution $A=0$ in the parameter plane $(\delta, \eta)$ for large delay times. The diagram shows the region of stability (gray), weak and strong instability (light gray and white, respectively).
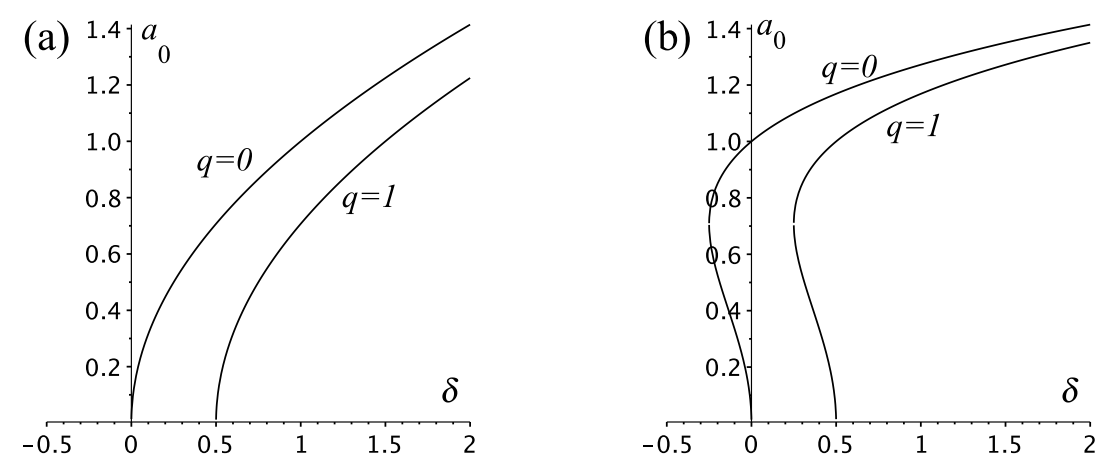

Fig. 4: Amplitude of plane wave solutions $a_{0}(\delta)$ versus parameter $\delta$ in the absence of the delay, given by Eqs. (15) and (14), for different spatial wavenumbers $q$. (a) Supercritical case, $\epsilon=-1, \mu=\nu=0$. (b) Subcritical case, $\epsilon=1, \mu=-1$, $\nu=-0.1$. In all plots $\beta=0.5$.

where

$$
a_{p}=a_{+} e^{i k x+\lambda t}+\bar{a}_{-} e^{-i k x+\bar{\lambda} t}
$$

is a small perturbation term with a growth rate $\lambda$. Here $\bar{\lambda}$ and $\bar{a}$ denote complex conjugation, and $k$ stands for different perturbation modes. Substitution of (16) into CGLE (1) with $\eta=0$ and linearization in $a_{p}$ yields

$$
\begin{array}{r}
\partial_{t} a_{p}+i \omega\left(a_{0}+a_{p}\right)=\left(\beta+\frac{i}{2}\right)\left(\partial_{x x} a_{p}+2 i q \partial_{x} a_{p}-q^{2}\left(a_{0}+a_{p}\right)\right)+\delta\left(a_{0}+a_{p}\right)+ \\
+(\epsilon+i)\left(a_{0}^{3}+2 a_{0}^{2} a_{p}+a_{0}^{2} \overline{a_{p}}\right)+(\mu+i \nu)\left(a_{0}^{5}+3 a_{0}^{2} a_{p}+2 a_{0}^{2} \overline{a_{p}}\right) .
\end{array}
$$

After substituting (17) into (18) and using Eq. (13) we obtain an equation involving two linearly independent functions $e^{i k x+\lambda t}$ and $e^{-i k x+\lambda t}$. Requiring that the coefficients at these functions are zero, we arrive at a system of linear equations for the unknowns $a_{-}$ and $a_{+}$:

$$
M\left(\begin{array}{l}
a_{+} \\
a_{-}
\end{array}\right)=0
$$


with

$$
M=\left[\begin{array}{cc}
\lambda+\left(\beta+\frac{i}{2}\right)\left(k^{2}+2 k q\right)- & -(\epsilon+i) a_{0}^{2}-2(\mu+i \nu) a_{0}^{4} \\
-(\epsilon+i) a_{0}^{2}-2(\mu+i \nu) a_{0}^{4} & \\
& \lambda+\left(\beta-\frac{i}{2}\right)\left(k^{2}-2 k q\right)- \\
-(\epsilon-i) a_{0}^{2}-2(\mu-i \nu) a_{0}^{4} & -(\epsilon-i) a_{0}^{2}-(\mu-i \nu) a_{0}^{4}
\end{array}\right] .
$$

Since we are looking for non-trivial solutions $\left(a_{+}, a_{-}\right)$, the characteristic equation for the perturbation growth rate $\lambda(k)$ is obtained by setting $\operatorname{det} M=0$ :

$$
\begin{array}{r}
\lambda^{2}+2\left(i k q+\beta k^{2}-\epsilon a_{0}^{2}-2 \mu a_{0}^{4}\right) \lambda-2\left((\nu+2 \mu \beta) k^{2}+2(\mu-2 \nu \beta) i k q\right) a_{0}^{4}+ \\
-\left((1+2 \epsilon \beta) k^{2}+2(\epsilon-2 \beta) i k q\right) a_{0}^{2}+\left(4 \beta^{2}+1\right)\left(k^{4} / 4+k^{2} q^{2}\right)=0 .
\end{array}
$$

Solutions $\lambda(k)$ can now be found explicitly and the maximum of their real parts determines the stability of plane waves. As it is seen from Fig. 5 the plane waves are stable for larger values of $\delta$ and become modulationally unstable with the decrease of $\delta$. This instability appears when the real part of the derivative $\partial_{k k} \lambda(0)$ changes its sign from negative to positive, see insets (a) and (b) in Fig. 5.

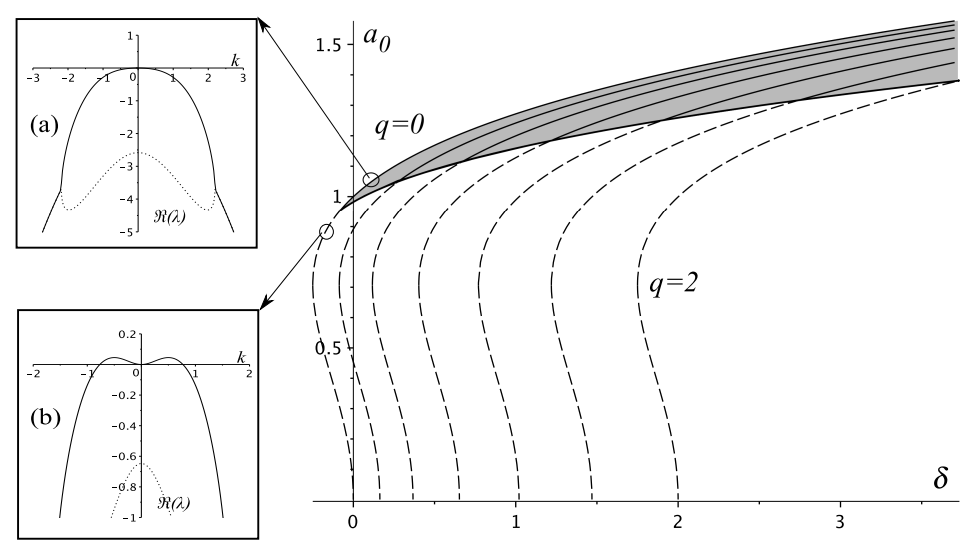

Fig. 5: Amplitude of plane wave solutions $a_{0}(\delta)$ in quintic CGLE (1) in the absence of the delay for different spatial wavenumbers $q$. Stability domain is shown in gray. Insets show the dependence of real part of the growth rate $\lambda(k)$ for selected plane waves. (a) $\delta=0.1, q=0, a_{0} \simeq 1.04$, stable case, (b) $\delta=-0.2, q=0, a_{0} \simeq 0.85$, unstable case. Other parameters: $\beta=0.5, \epsilon=1, \mu=-1$, and $\nu=-0.1$.

The threshold of the modulational instability is given by the condition $\Re\left[\partial_{k k} \lambda(0)\right]=0$. For small $k$ (long-wavelength limit) the Taylor expansion of $\lambda(k)$ reads

$$
\lambda(k)=\left(-\frac{C_{3}}{C_{1}}-q\right) i k+\left(-\frac{C_{3}^{2}}{128 C_{1}^{3}}-\frac{C_{2}^{2}}{C_{1}}-\beta\right) k^{2}+\mathcal{O}\left(k^{3}\right),
$$

where $C_{1}=\epsilon a_{0}^{2}+2 \mu a_{0}^{4}, C_{2}=16 \beta^{2} q^{2}+4 a_{0}^{2}+8 \nu a_{0}^{4}$ and $C_{3}=64 \beta^{3} q^{3}-4 \beta q C_{2}$. In particular, the stability boundary $\partial_{k k} \lambda(0)=0$ is given by

$$
\frac{C_{3}^{2}}{128 C_{1}^{3}}+\frac{C_{2}^{2}}{C_{1}}+\beta=0 .
$$

Figure 5 shows the amplitude $a_{0}$ of plane wave solutions versus $\delta$ for different wavenumbers $q$ along with the examples of $\Re[\lambda(k)]$ for stable an unstable cases respectively. Stable solutions are depicted by solid lines and the stable domain is shown in gray. The first 
zero wavenumber plane wave solution $A=a_{0} e^{i \omega t}$ with $q=0, a_{0}^{2}=\left[-\epsilon \pm \sqrt{\epsilon^{2}-4 \mu \delta}\right] / 2 \mu$, and $\omega=a_{0}^{2}+\nu a_{0}^{4}$ is stable for

$$
\delta>\frac{4 \beta \epsilon(\beta \epsilon+2 \beta \mu+\nu+1)+2(2 \beta \mu+\nu+1)}{4(2 \beta \mu+\nu)^{2}} .
$$

With the further increase of $\delta$, stable plane wave solutions with $q^{2}>0$ appear.

\subsection{Case of delay $\tau$}

\section{Description of the set of plane wave solutions}

Substituting $A=a_{0} e^{i q x+i \omega t}$ into CGLE with delayed feedback (1) we obtain the equation connecting the amplitude $a_{0}$, frequency $\omega$, and wavenumber $q$ of the plane waves

$$
i \omega=-\left(\beta+\frac{i}{2}\right) q^{2}+\delta+(\epsilon+i) a_{0}^{2}+(\mu+i \nu) a_{0}^{4}+\eta e^{i \varphi} e^{-i \omega \tau} .
$$

After separating real and imaginary parts of (24), we obtain

$$
\begin{aligned}
0 & =-\beta q^{2}+\delta+\epsilon a_{0}^{2}+\mu a_{0}^{4}+\eta \cos (\omega \tau-\varphi), \\
\omega & =-\frac{q^{2}}{2}+a_{0}^{2}+\nu a_{0}^{4}-\eta \sin (\omega \tau-\varphi) .
\end{aligned}
$$

In the coordinates $\left(\delta, \omega, a_{0}\right)$, the set of solutions of Eqs. 25-26) for each $q$ lies on the surface of a tube, which is implicitly defined by the following equation

$$
\left(-\beta q^{2}+\delta+\epsilon a_{0}^{2}+\mu a_{0}^{4}\right)^{2}+\left(\omega+\frac{q^{2}}{2}-a_{0}^{2}-\nu a_{0}^{4}\right)^{2}=\eta^{2}
$$

(see Fig. 66), obtained from Eqs. 25 - 26) by exclusion of $\omega \tau-\varphi$. For fixed parameters, including $\delta$, this is a one-dimensional set, see the cross-section in Fig. 6.

Solving (25) with respect to $a_{0}^{2}$ and substituting the result into (26), we obtain the equation for the frequencies $\omega$ of the plane waves

$$
\begin{array}{r}
0=f_{ \pm}(\omega)=\omega+\frac{q^{2}}{2}+\frac{\epsilon \pm \sqrt{\epsilon^{2}-4 \mu\left(\delta-\beta q^{2}+\eta \cos (\omega \tau-\varphi)\right)}}{2 \mu}- \\
-\frac{\nu\left(\epsilon \pm \sqrt{\epsilon^{2}-4 \mu\left(\delta-\beta q^{2}+\eta \cos (\omega \tau-\varphi)\right)}\right)^{2}}{4 \mu^{2}}+\eta \sin (\omega \tau-\varphi)
\end{array}
$$

which can be studied numerically for any fixed value of the delay $\tau$. The functions $f_{ \pm}(\omega)$ are shown on Fig. 7. In particular, with the increase of $\tau$ the functions $f_{ \pm}(\omega)$ oscillate faster and number of solutions grows. This corresponds to a general result obtained in [33] stating that in the limit of large delay the number of periodic solutions increases linearly with $\tau$. Eventually the solutions fill the curves defined by relation (27). Figure (8) shows the exact solutions $\left(a_{0}, \omega\right)$ for increasing delay time $\tau$ as points on these curves. It is interesting to remark the strong analogy between the curve (27) of the plane wave solutions and the ellipse of external cavity modes appearing in rate equation models for semiconductor lasers with time delayed feedback, where the notion is frequently used $[18,11,34,25,22]$. 


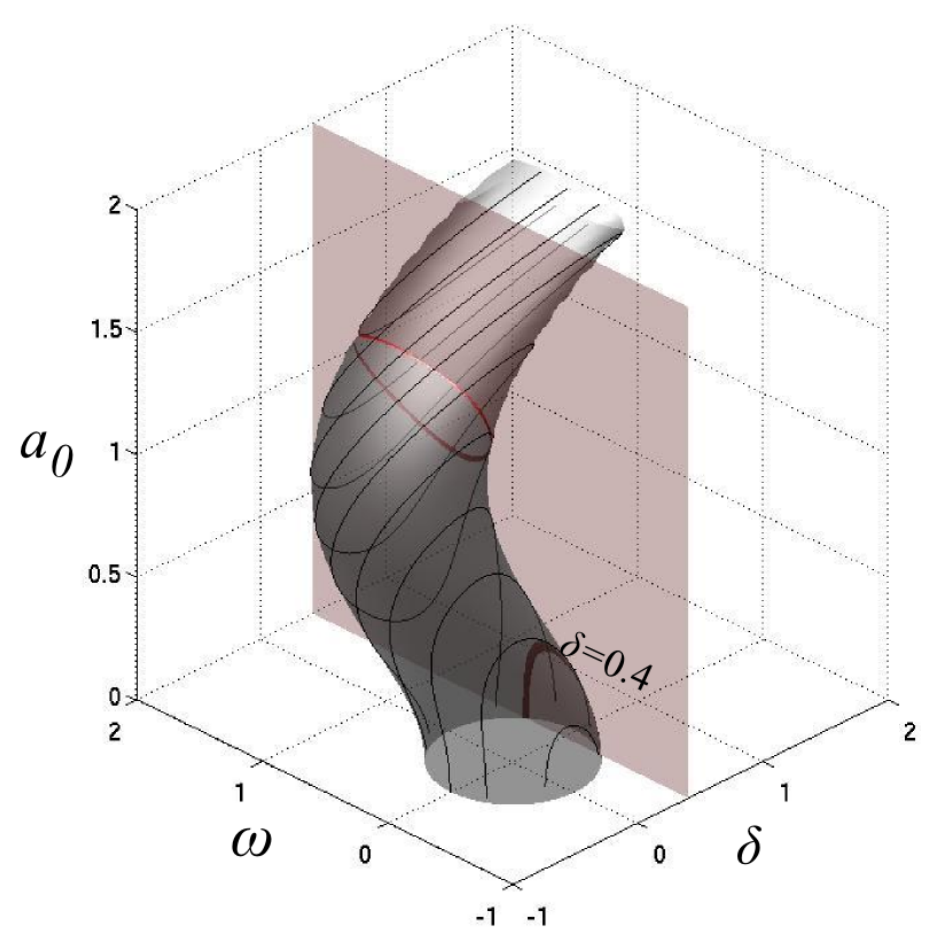

Fig. 6: Amplitudes $a_{0}$ and frequencies $\omega$ of plane wave solutions as a function of $\delta$, defined by Eq. (27). Black curve indicates the branch of plane wave solutions (defined by (25)-(26)) for $\tau=25$ and $\varphi=0$. Other parameters: $q=0, \eta=0.5, \beta=0.5$, $\epsilon=1, \mu=-1$, and $\nu=-0.1$. Red curve shows the cross-section of the tube by the plane of the fixed parameter $\delta=0.4$.

Let us consider the effect of the feedback phase $\varphi$, which shifts the function $f_{ \pm}(\omega)$, see Fig. 7. For small delay times, when $f_{ \pm}(\omega)$ oscillates slowly, the role of $\varphi$ is pronounced since a few of individual solutions are moved along the curve $a_{0}(\omega)$ and new solutions can be born. As $\tau$ increases, the overall number of plane wave solutions becomes large, thus diminishing the effect of the feedback phase, as shown in Figs. 7(b) and 8(b).

Even though exact values of $a_{0}$ and $\omega$ for any fixed set of parameters can only be computed numerically, the branches of plane wave solutions versus parameter $\delta$ can be obtained explicitly in a parametric form $\left(a_{0}(\omega), \delta(\omega)\right)$. Namely, the amplitude $a_{0}(\omega)$ is the solution of Eq. (26). Substituting $a_{0}(\omega)$ into Eq. (25) and solving the resulting equation for $\delta$, we obtain an expression for $\delta(\omega)$. As a result, the branches of plane waves have the parametric form

$$
\begin{array}{cc}
a_{0}(\omega): & =\left\{\text { solution of } \nu\left(a_{0}^{2}\right)^{2}+a_{0}^{2}-\eta \sin (\omega \tau-\varphi)-\omega-\frac{q^{2}}{2}=0\right\} \\
\delta(\omega): & =\beta q^{2}-\epsilon a_{0}^{2}(\omega)-\mu a_{0}^{4}(\omega)-\eta \cos (\omega \tau-\varphi) .
\end{array}
$$

The branches of plane wave solutions obtained using this procedure are shown in Fig. 9 and Fig. 10 for cubic and quintic CGLE, respectively. Interestingly, the branches have the form of snaking curves, which are constrained between two "limiting" branches (14) (red and blue curves in Figs. 9 10), which can be obtained by setting $\tau=0, \varphi=0$ and $\tau=0$, $\varphi=\pi$, respectively. One can see that the increase of $\tau$ leads to even more dense snaking of the curve (Fig. 9(b)), whereas the increase of $q$ shifts the branches to the right. 

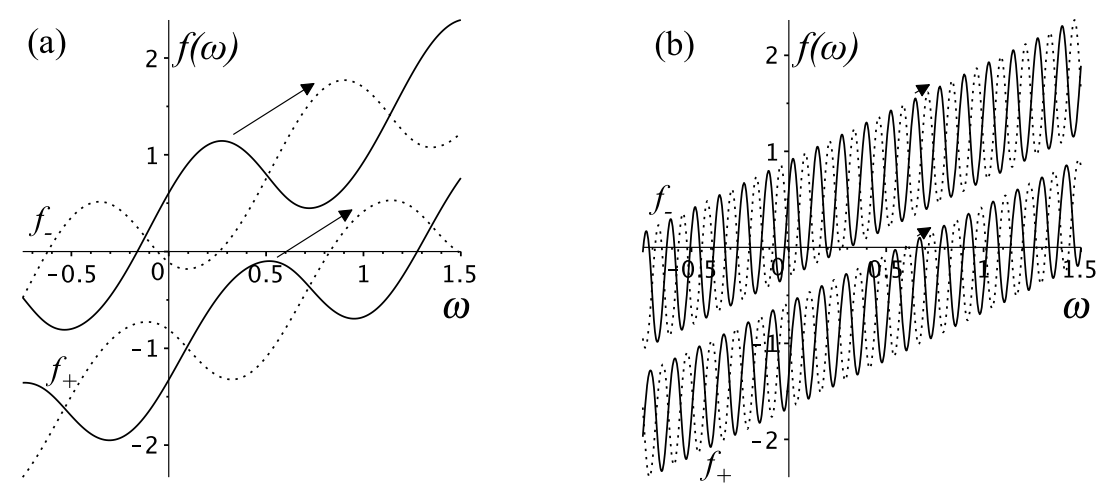

Fig. 7: Zeros of functions $f_{ \pm}(\omega)$ determine frequencies $\omega$ of plane waves. $f_{ \pm}(\omega)$ are calculated from Eq. (28) for (a) $\tau=5$ and (b) $\tau=50$. Solid lines: $\varphi=0$, dashed lines: $\varphi=\pi$. Other parameters: $q=0, \delta=0.4, \eta=0.5, \beta=0.5, \epsilon=1, \mu=-1$, and $\nu=-0.1$.
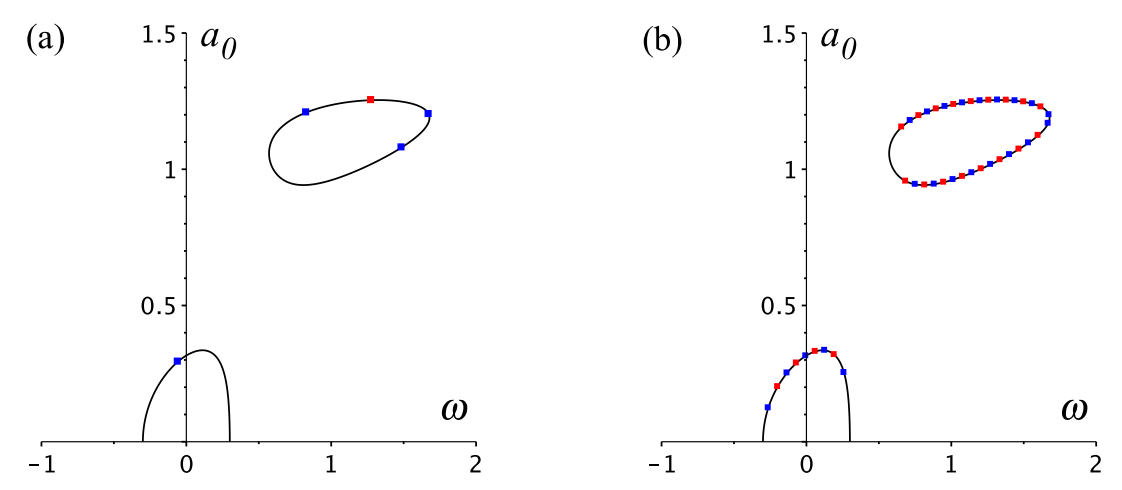

Fig. 8: Amplitudes $a_{0}$ and frequencies $\omega$ of plane wave solutions for fixed $\delta=0.4$. The solutions are located on curves determined by Eq. (27) (solid lines), which are independent of the delay $\tau$. Exact plane wave solutions for different values of $\tau$ are shown by points: (a) $\tau=5$, (b) $\tau=50$. Red points: $\varphi=0$, blue points: $\varphi=\pi$. Other parameters: $q=0, \eta=0.5, \beta=0.5, \epsilon=1, \mu=-1$, and $\nu=-0.1$.

\section{Stability of plane wave solutions}

As in the case without delayed feedback, we use the ansatz (16) to investigate the stability of plane waves. After substituting it into delayed CGLE (1) and linearization in $a_{p}$, we obtain:

$$
\begin{array}{r}
\partial_{t} a_{p}+i \omega\left(a_{0}+a_{p}\right)=\left(\beta+\frac{i}{2}\right)\left(\partial_{x x} a_{p}+2 i q \partial_{x} a_{p}-q^{2}\left(a_{0}+a_{p}\right)\right)+\delta\left(a_{0}+a_{p}\right)+ \\
+(\epsilon+i)\left(a_{0}^{3}+2 a_{0}^{2} a_{p}+a_{0}^{2} \overline{a_{p}}\right)+(\mu+i \nu)\left(a_{0}^{5}+3 a_{0}^{2} a_{p}+2 a_{0}^{2} \overline{a_{p}}\right) \\
+\eta e^{i \varphi-i \omega \tau}\left(a_{0}+a_{p}(t-\tau)\right) .
\end{array}
$$

We simplify this equation using Eq. 24) and substitute the exponential ansatz (17) for $a_{p}(x, t)$ into it. As a result, similarly to Section 3.1. we obtain a linear system of equations with respect to two unknowns $a_{+}$and $a_{-}$:

$$
M_{\tau}\left(\begin{array}{c}
a_{+} \\
a_{-}
\end{array}\right)=0
$$



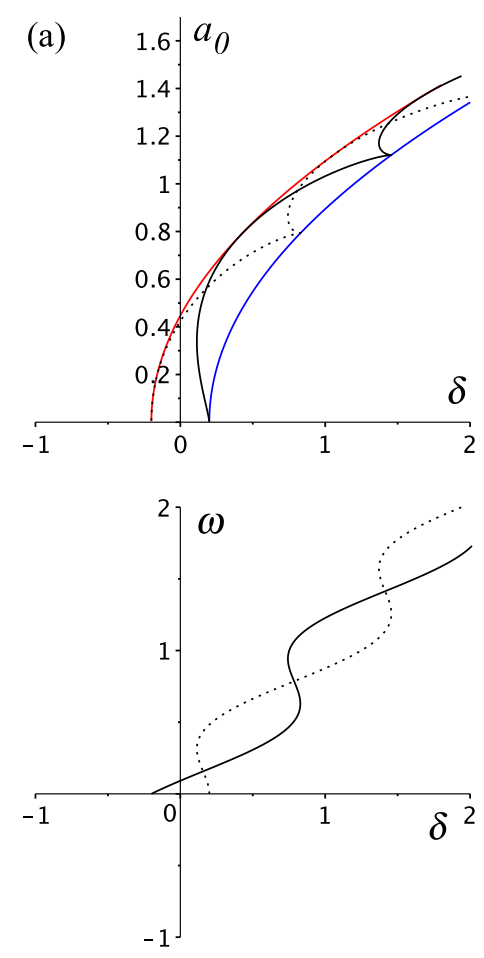
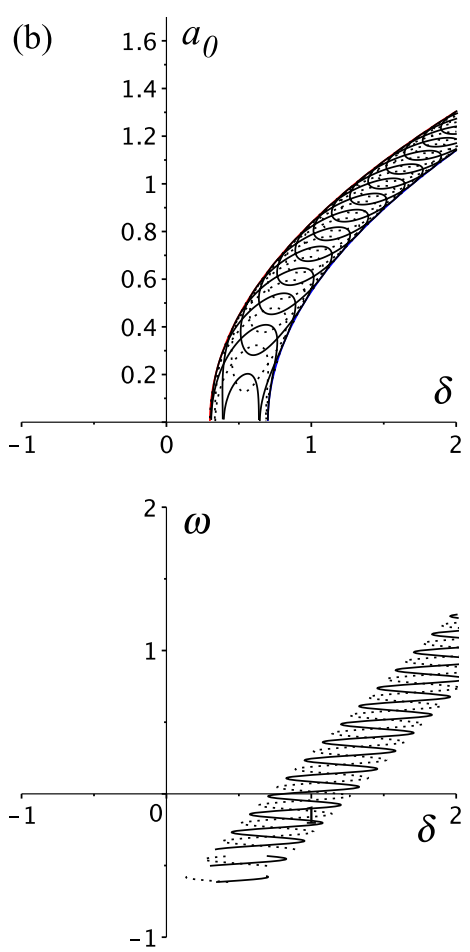

Fig. 9: Amplitudes $a_{0}(\delta)$ and frequencies $\omega(\delta)$ of plane wave solutions in cubic CGLE with delayed feedback for (a) $\tau=5, q=0$, (b) $\tau=50, q=1$. Solid black lines: $\varphi=0$, dotted lines: $\varphi=\pi$. The enveloping red and blue lines are the branches of plane waves solutions for $\tau=0, \varphi=\pi$ and $\tau=0, \varphi=0$, respectively. Other parameters: $\eta=0.2, \beta=0.5, \epsilon=1, \mu=\nu=0$.

where

$$
M_{\tau}=\left[\begin{array}{cc}
\lambda+i \omega-\delta+\left(\beta+\frac{i}{2}\right)\left(k^{2}+2 k q+q^{2}\right)- & -(\epsilon+i) a_{0}^{2}-2(\mu+i \nu) a_{0}^{4} \\
-2(\epsilon+i) a_{0}^{2}-3(\mu+i \nu) a_{0}^{4}-\eta e^{-\lambda \tau} e^{i \varphi-i \omega \tau} & \\
& \lambda-i \omega-\delta+\left(\beta-\frac{i}{2}\right)\left(k^{2}-2 k q+q^{2}\right)- \\
-(\epsilon-i) a_{0}^{2}-2(\mu-i \nu) a_{0}^{4} & -2(\epsilon-i) a_{0}^{2}-3(\mu-i \nu) a_{0}^{4}-\eta e^{-\lambda \tau} e^{-i \varphi+i \omega \tau}
\end{array}\right] .
$$

The condition

$$
\operatorname{det} M_{\tau}=0
$$

now gives us the characteristic equation for the perturbation growth rate $\lambda$.

Stability of individual plane wave solutions with arbitrary delay time $\tau$ is determined by the real parts of the roots of the characteristic equation $(32)$. If for all $k$, the roots satisfy $\Re[\lambda]<0$ (except the trivial one with $\Re[\lambda]=0$ ), then the plane wave is stable. For a fixed $q$, the branch of plane waves is given parametrically by $\left(a_{0}(\omega), \delta(\omega)\right)$, defined by Eq. (29). Substituting $a_{0}(\omega)$ and $\delta(\omega)$ in Eq. (32), we obtain a nonlinear characteristic equation for $\lambda$

$$
\mathcal{F}(\lambda ; q, \omega, k, \mathbf{p})=0,
$$

where $\mathbf{p}$ denotes system parameters $(\beta, \epsilon, \mu, \nu, \eta, \varphi, \tau)$. Equation (33) was solved numerically for fixed values of $\mathbf{p}$ and varying $\omega$. In this way we obtain the stability properties for the parametrically defined family of plane waves $\left(a_{0}(\omega), \delta(\omega)\right)$ for the given CGLE parameters and wavenumber $q$. Figure 11 shows the stability properties on the branches of plane waves $\left(a_{0}(\omega), \delta(\omega)\right)$ with $q=0$ and various delay times $\tau$. Stable solutions are plotted in green, unstable in red. Figure 11(a) illustrates the effect of the feedback 
(a)
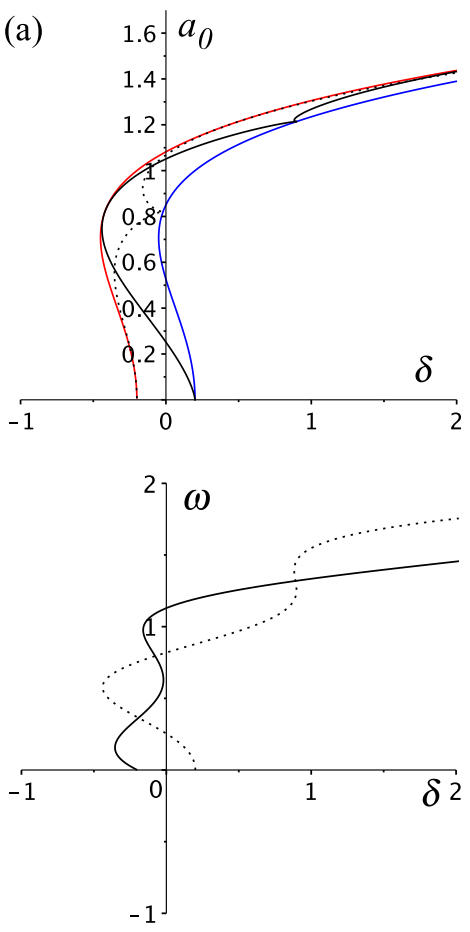

(b)
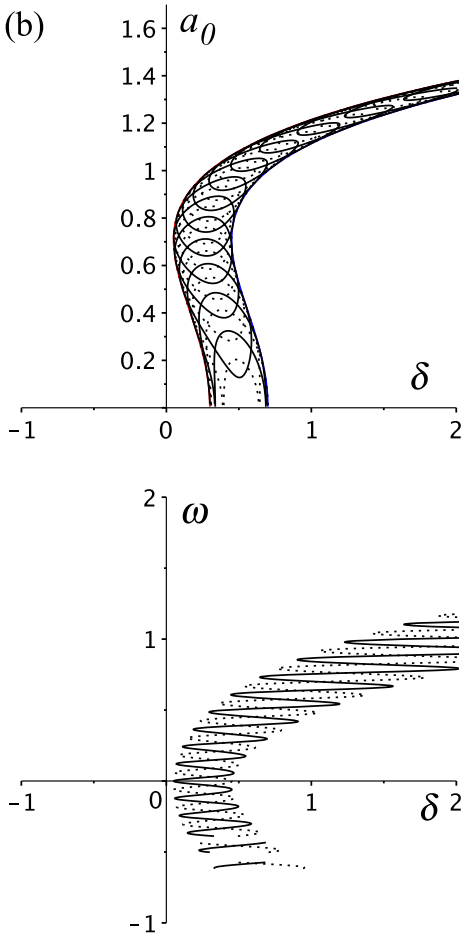

Fig. 10: Amplitudes $a_{0}(\delta)$ and frequencies $\omega(\delta)$ of plane wave solutions in quintic CGLE with delayed feedback for (a) $\tau=5, q=0$, (b) $\tau=50, q=1$. Solid black lines: $\varphi=0$, dotted lines: $\varphi=\pi$. The enveloping red and blue lines are the branches of plane wave solutions for $\tau=0, \varphi=\pi$ and $\tau=0, \varphi=0$, respectively. Other parameters: $\eta=0.2, \beta=0.5, \epsilon=1, \mu=-1, \nu=-0.1$.

phase $\varphi$ on plane wave solutions and their stability at relatively small delay time $\tau=5$, whereas Fig. 11(b) depicts the bifurcations for larger delay time $\tau=50$. One can observe the growing number of multistable plane waves with the increase of the delay. With the increase of the parameter $\delta$ high amplitude parts of the snaking branches with the higher amplitude becomes stable while low amplitude parts remain unstable. An additional analytical insight into the structure of stable and unstable regions is obtained by using the large delay approximation, which is discussed in the next section.

\subsection{Large delay}

For large delay times, the plane wave solutions fill the tube defined by Eq. (27) densely, see also Figs. 6 and 8(b). Hence, instead of looking at individual solutions and solving the transcendental Eq. (28), it is convenient to parametrize the whole family of solutions by a parameter $\theta=(\omega \tau-\varphi+\pi) \bmod 2 \pi$, which may be represented as an angular coordinate on the tube. Every single solution for given control parameters and wavevector $q$ can be uniquely defined by the coordinate $\theta$. Therefore, one can consider the question about the stability of a plane wave at a given $\theta$-value with the amplitude $a_{0}(\theta)$ and the frequency $\omega(\theta)$. The growth rate $\lambda$, which determines the stability of an individual plane wave solution, is obtained from the characteristic equation depending just on the system parameters and the coordinate $\theta$. Note that $\theta=\pi$ corresponds to the plane waves with the maximal amplitude $a_{0}$. The two sides of the tube of plane wave solutions, one with $\theta<\pi$ and another with $\theta>\pi$, are projected onto the same set in the $\left(\delta, a_{0}\right)$ plane, but 

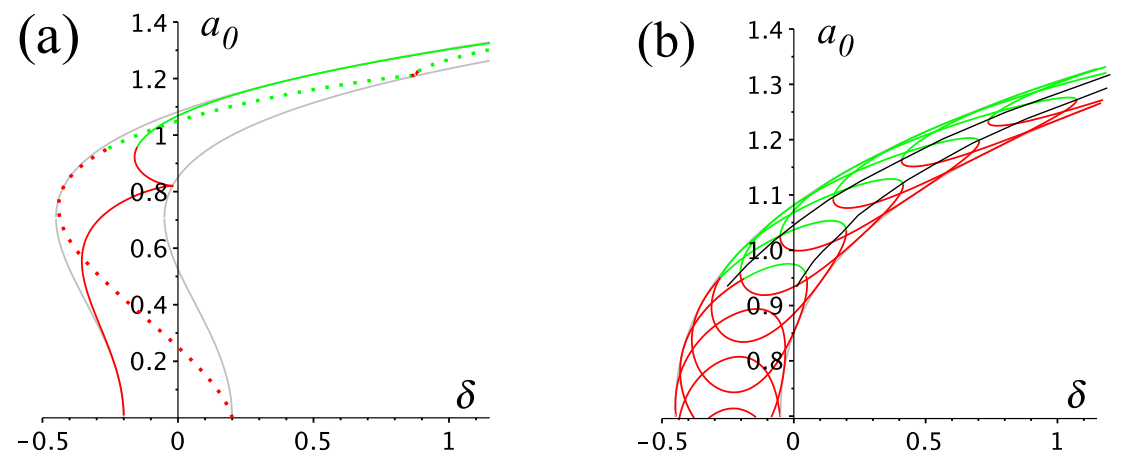

Fig. 11: Stability of the branches of plain wave solutions for different delay times (a) $\tau=5, \varphi=0$ (solid line), $\varphi=\pi$ (dotted line) (b) $\tau=50, \varphi=0$. Stable parts are shown in green and unstable - in red. Black lines in (b) denote the destabilization borders in the limit of large delay. Other parameters: $\eta=0.2, \beta=0.5, \epsilon=1$, $\mu=-1$, and $\nu=-0.1$.

correspond to different values of $\omega$.

To study the strong instability of plane wave solutions, again, as in Sec. 3.3, we neglect the terms containing $e^{-\lambda \tau}$ in Eq. (32). This gives us a quadratic equation for $\lambda$. For a given parameter $\theta$, the plane wave is strongly unstable, if the maximum of $\Re[\lambda(k, \theta)]$ is positive. Figure 12 shows the the real part of $\lambda(k, \theta)$ for reduced equation (32) for two different values of the wavenumber, $q=0$ and $q=1$ ( $\delta$ is fixed to 0.5). Red curves depict zero level lines. We observe, that at larger values of $q$, unlike the feedback-free case, the destabilization occurs first at non-zero values of the perturbation wavenumber $k$.
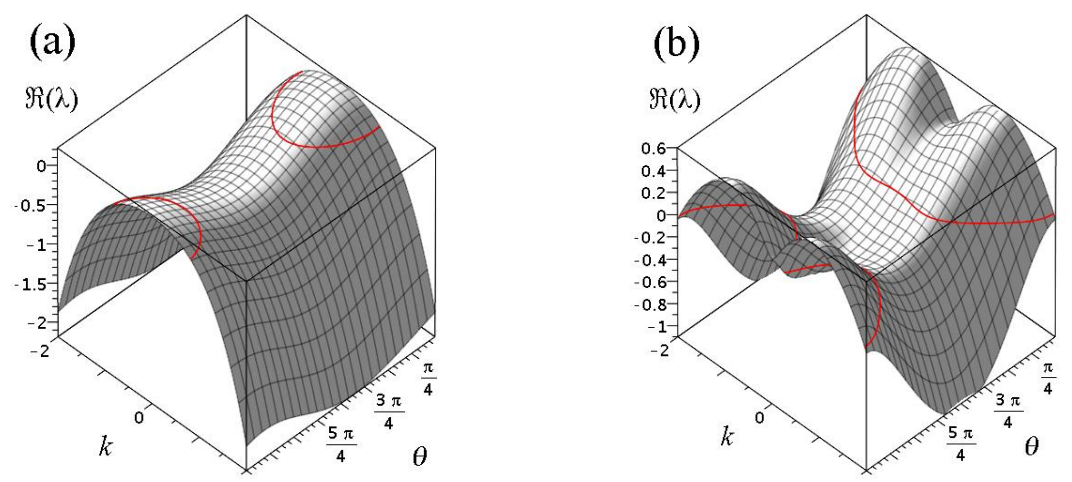

Fig. 12: Real parts of the eigenvalues $\Re[\lambda(k, \theta)]$ describing the strong instability of plane waves. For a given plane wave, which is determined by the parameter $\theta$, the positiveness of $\Re[\lambda(k, \theta)]$ for some $k$ implies the strong instability. Red curves depict zero level lines. (a) $q=0$, (b) $q=1$. On panel (a), the plane waves are strongly unstable for $\theta \lesssim 1.9$ and $\theta \gtrsim 5.0$. On panel (b), the plane waves are strongly unstable for $\theta \lesssim 1.9$ and $\theta \gtrsim 4.5$. Other parameters are: $\delta=0.5, \eta=0.2$, $\beta=0.5, \epsilon=1, \mu=-1$, and $\nu=-0.1$.

In order to determine the strong instability boundary of the plane wave solutions, in Fig. 13 we plot on $(\delta, \theta)$ plane zero contour levels $\Re[\lambda(\delta, \theta)]=0$ corresponding to different perturbation wavenumbers $k$. Figure 13 (a) shows that for the plane wave with $q=0$, stability border almost coincides with the zero level line corresponding to $k=0$, depicted by the black line. By contrast, for the plane wave with $q=1$ a significant part of stability 
border, shown in red color, corresponds to destabilization with non-zero perturbation wavenumber $k \sim 0.9$ (see Fig. 13(b)).
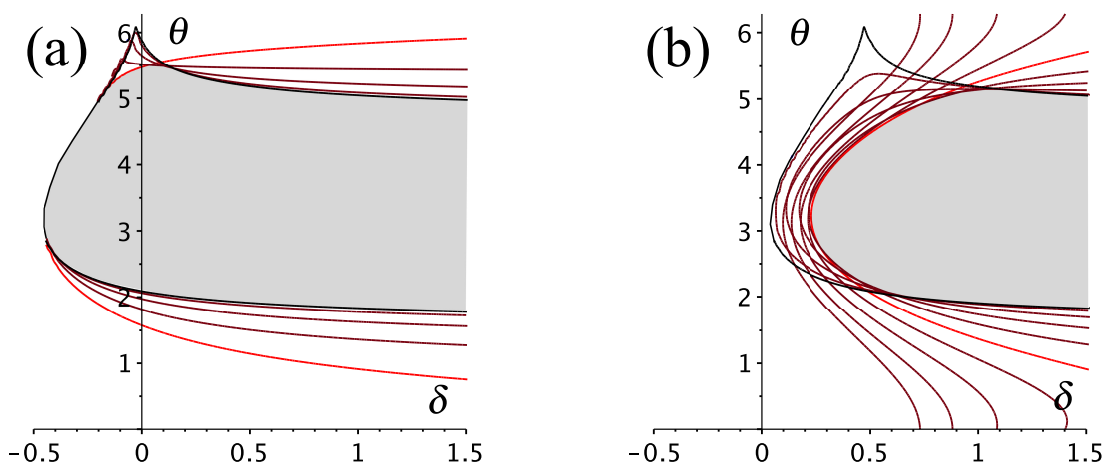

Fig. 13: Strong instability of the plane wave solutions with $q=0$ (a) and $q=1$ (b). Gray region shows the absence of strong instability. Different curves indicate zero contour levels of $\Re[\lambda(\delta, \theta)]$ corresponding to different perturbation wavenumbers $k$. (a) $q=0$, (b) $q=1$. Other parameters: $\eta=0.2, \beta=0.5, \epsilon=1, \mu=-1$, and $\nu=-0.1$.

In the limit of large delay, the weak instability boundary is determined by the pseudocontinuous spectrum

$$
\lambda=\frac{\gamma}{\tau}+i \xi
$$

To calculate this spectrum we substitute (34) into Eq. (32) and neglect the terms proportional to $\gamma / \tau$. Then, denoting

$$
Y=e^{-\gamma} e^{-i \xi \tau}
$$

one can solve the resulting quadratic equation with respect to $Y$. The two roots $Y_{1}$ and $Y_{2}$ of this equation depend on the model equation parameters $\mathbf{p}$, plane wave $(q, \theta)$, wavenumber of the perturbation $k$, and the delay induced perturbation modes $\xi$. For brevity we do not write down the explicit form of these solutions. The factor $\gamma$ in (35) is given by

$$
\gamma_{1,2}=-\ln \left|Y_{1,2}(k, \xi ; q, \theta, \mathbf{p})\right| .
$$

For every plane wave solution defined by $q$ and $\theta$ and fixed parameters $\mathbf{p}$ we obtain two surfaces $\gamma_{1,2}(k, \xi)$, which generalize the dispersion relation (see Sec. (2.3)). If $\gamma_{1,2}<0$ for all wavenumbers $k$ and delay modes $\xi$ (except the trivial eigenvalue corresponding to the Goldstone mode), then the corresponding plane wave is stable (provided no strong instability exists). Otherwise, it is weakly unstable. Thus, the weak instability of the plane wave solutions is determined by considering the behavior of the two surfaces $\gamma_{1,2}(k, \xi)$, or, more specifically, the upper one.

Figure (14) shows the upper branch of $\gamma_{1,2}(k, \xi)$ calculated for different values of $\theta, \delta$, and $q$. Even though the surfaces $\gamma(k, \xi)$ are given by explicit expressions, we were not able to find simple analytical conditions for weak instability in terms of $\theta$ and $q$. Instead, we determined the $\operatorname{sign}$ of $\sup [\gamma(k, \xi)]$ numerically. The resulting bifurcation diagrams showing the regions of stable, weakly unstable, and strongly unstable plane waves with $q=0$ and $q=1$ on $(\delta, \theta)$ plane are presented in Figure (15).

The corresponding bifurcation diagrams in $\left(\delta, a_{0}\right)$ plane are shown in Figs. 16 and 17. Note that in contrast to the unique parameterization of the plane waves with the 

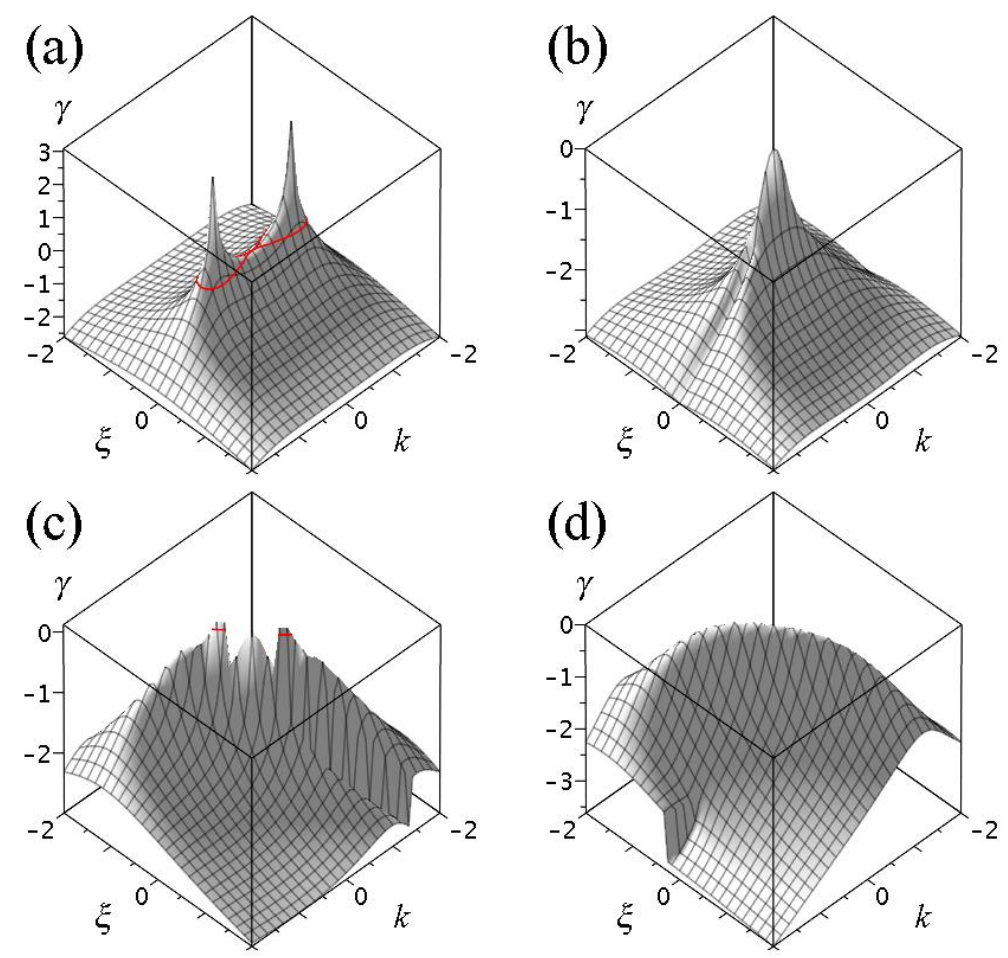

Fig. 14: The rescaled growth rate $\gamma=\tau \Re[\lambda]$ of different perturbation modes (generalized dispersion relation) for different plane wave solutions. $k$ is the spatial wavenumber of the perturbations, $\xi$ stands for the delay induced modes. The positiveness of $\gamma$ implies weak instability of the plane wave. The surface $\gamma(\xi, q)$ is obtained from Eq. (36) for different values of $\theta, \delta$, and $q$. (a) $\theta=0, \delta=0.5, q=0$. (b) $\theta=2, \delta=1.0, q=0$. (c) $\theta=2, \delta=1.0, q=1$. (d) $\theta=4, \delta=1.0, q=1$. Panels (b) and (d) [(a) and (c)] correspond to stable [weakly unstable] plane waves. Other parameters: $\eta=0.2, \beta=0.5, \epsilon=1, \mu=-1$, and $\nu=-0.1$.

parameter $\theta$, in general the plane wave solution is not uniquely defined by the parameter $a_{0}$ (the frequencies $\omega$ can still be different for the same $a_{0}$ ), which means that the sets with $0 \leq \theta \leq \pi$ and $\pi \leq \theta \leq 2 \pi$ are overlapping on the $\left(\delta, a_{0}\right)$ plane. Moreover, the stability properties of these two sets are not symmetric with respect to $\theta=\pi$, as shown in Figs. 16 17(a) and (b), respectively.

Note that the areas of weak instability for quintic CGLE in the large delay limit are consistent with the stability borders obtained numerically for $\tau=50$ (compare the transitions from stability to instability on the branches of solutions and black dashed lines determining the boundaries in the large delay limit in Fig. 11(b)). It provides also a simple qualitative way how to predict the appearance of stable plane waves: namely, when the branches of plane waves for a finite $\tau$ appear to be in the domain of stability given in Figs. 16 17 (dark grey shaded), then they are likely to be stable. Since the domains are independent on $\tau$, the number of coexisting stable plane waves grows linearly with $\tau$ [33].

\subsection{Numerical simulations}

To investigate the behavior of particular plane wave solutions, e.g. the onset of destabilization and convergence to stable solutions, we performed direct numerical integration of the delayed quintic CGLE (1). An embedded adaptive Cash-Carp scheme for time- 

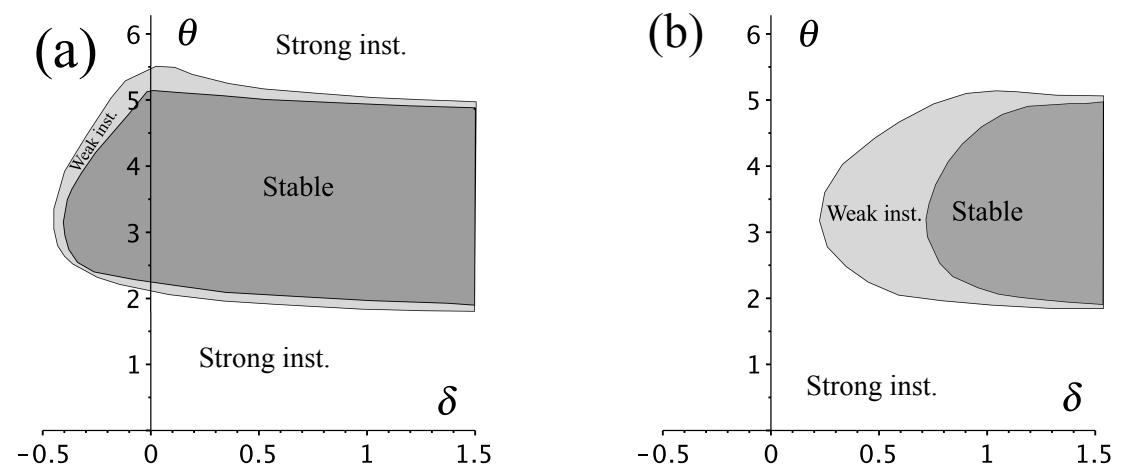

Fig. 15: Regions of stability (dark gray), weak (light gray) and strong (white) instability for the plane waves in quintic CGLE on $(\delta, \theta)$ plane. (a) $q=0$, (b) $q=1$. Other parameters: $\eta=0.2, \beta=0.5, \epsilon=1, \mu=-1$, and $\nu=-0.1$.
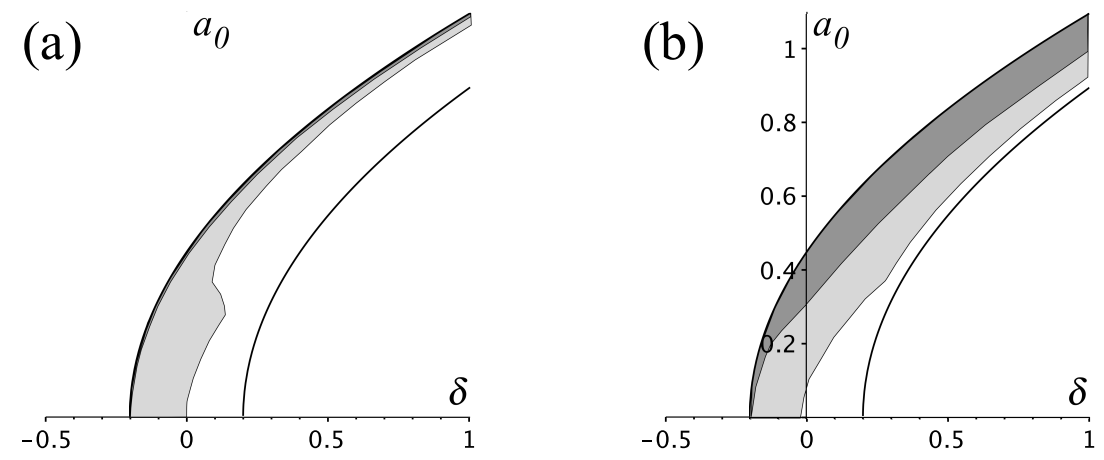

Fig. 16: Regions of stability (dark gray), weak (light gray) and strong (white) instability for the plane waves in cubic CGLE in $\left(\delta, a_{0}\right)$ plane. (a) $\theta<\pi$, (b) $\theta>\pi$. Other parameters $q=0, \eta=0.2, \beta=0.5$, and $\epsilon=-1$.

stepping was used with the spatial derivative was treated by three-point central finite difference scheme. Periodic boundary conditions were are applied, and the length of the system is chosen to include 16 spatial periods of simulated plane waves with $q=1$. Space was discretized into 500 points, while relative tolerance for Cash-Karp scheme was set at $10^{-6}$.

First, we considered the solution starting in the vicinity of the unstable homogeneous plane wave with $q=0$, see red point in Fig. 18(b). Fig. 18(a) shows the evolution of the amplitude, while Fig. 18(b) shows the stable plane wave (green circle), to which the solution is attracted. In the case when there are no stable plane waves for given system parameters, the solution converges to the stable homogeneous state $A=0$.

Transitions similar to those shown in Fig. 18 were also observed for $q \neq 0$, see Fig. 19. where two unstable plane waves with $q=1$ were chosen as initial conditions. Figures 20 (a) and (b) present the spatio-temporal evolution of the solutions corresponding to the transitions shown in Fig. 19. It is seen that the solution (a) develops defects after several delay cycles and transforms into a slightly modulated solution with lower spatial wavenumber $(q=0.25)$, which is stable for the given control parameter values. Note that for these parameter values there are no stable plane waves with the wavenumber $q=1$. By contrast, solution (b) does not change its principal spatial wavenumber. After several delay periods of transient it converges without defects to a stable plane wave having the same wavenumber $q=1$, see corresponding transition (b) in Fig. 19 . 

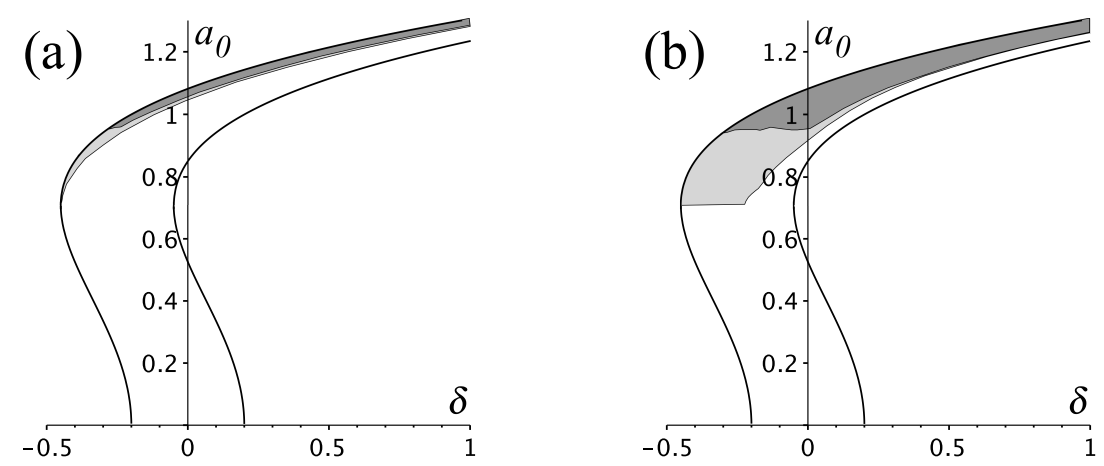

Fig. 17: Regions of stability (dark gray), weak (light gray) and strong (white) instability for the plane waves in quintic CGLE in $\left(\delta, a_{0}\right)$ plane. (a) $\theta<\pi$, (b) $\theta>\pi$. Other parameters $q=0, \eta=0.2, \beta=0.5, \epsilon=1, \mu=-1$, and $\nu=-0.1$.
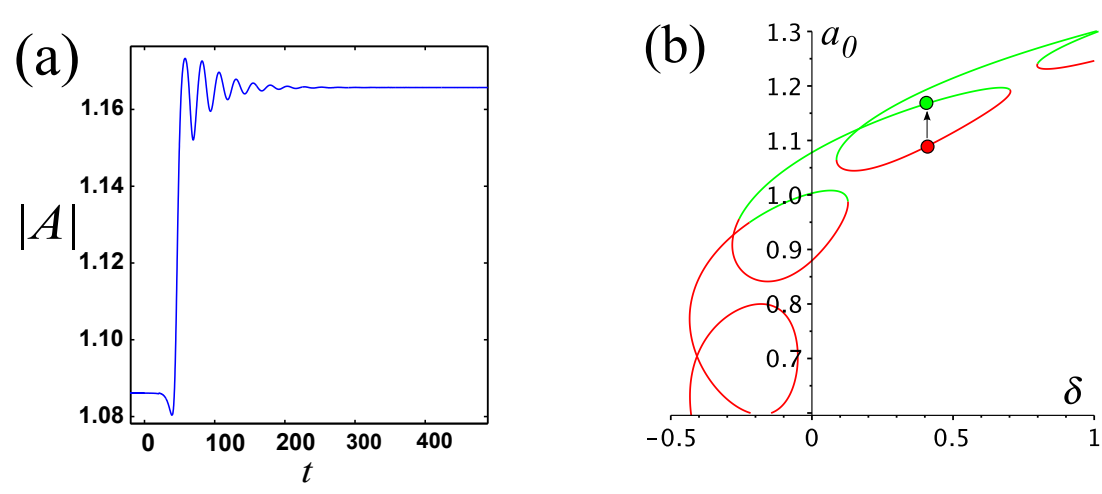

Fig. 18: Solution starting from the neighborhood of an unstable plane wave with $q=0$ is attracted to a stable plane wave with the same wavenumber. (a) depicts the time evolution of the amplitude $a$. (b) shows the corresponding transition in $\left(\delta, a_{0}\right)$ plane. Initial unstable solution: $\omega=1.12, a_{0}=1.086$ (red circle). Resulting stable solution: $\omega=0.998, a_{0}=1.166$ (green circle). System parameters: $\delta=$ $0.023, \tau=20, \eta=0.2, \beta=0.5, \epsilon=1, \mu=-1$, and $\nu=-0.1$.

Now we choose $\tau$ sufficiently large $(\tau=50)$, so that we can exploit the results of asymptotic analysis obtained in the limit of large delay. We consider a plane wave with the control parameters from weakly unstable region on $(\theta, \delta)$ plane and investigate the evolution of a small perturbation of this plane wave. We expect to observe the onset of destabilization after a long period. Figure 21(a) illustrates the choice of the initial plane wave, while Fig. 21(b) shows the transient and the onset of destabilization. The solution stays close to the weakly unstable plane wave for about 80 delay time periods, and then goes away from it. Eventually, the solution is attracted to a stable plane wave with $q=1 / 8$.

\section{Conclusions}

We have investigated the properties of plane wave solutions of cubic and cubic-quintic CGLE with delayed feedback. It is demonstrated that the delayed feedback induces a multistability of plane wave solutions with the same wavenumber $q$. As the gain parameter $\delta$ is varied, the branches of plane wave solutions are shown to exhibit a snaking 


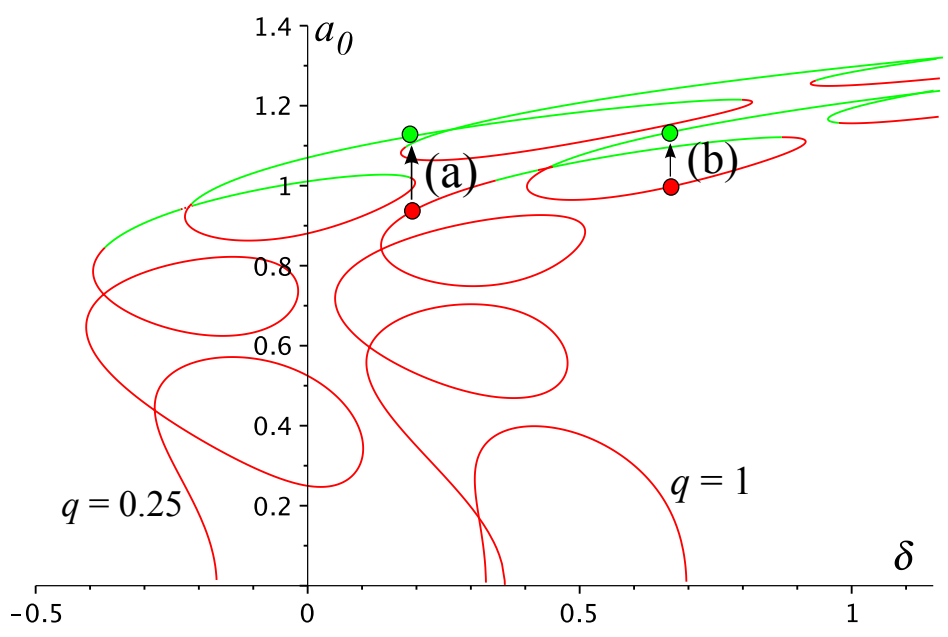

Fig. 19: Evolution of solutions starting close to unstable plane waves with $q=1$. Solution (a) with $\delta=0.19, \omega=0.31$, and $a_{0}=0.87$ approach the stable plane wave with different wavenumber $q=0.25$, and $\omega=0.969, a_{0}=1.12$. Solution (b) $\delta=0.66$, $\omega=0.5, a_{0}=0.99$ approaches the stable solution with the same wavenumber $q=1$, and $\omega=0.626, a_{0}=1.11$.

(a)

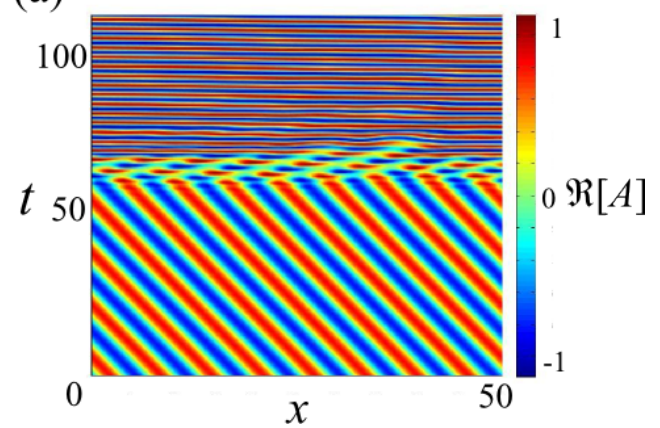

(b)

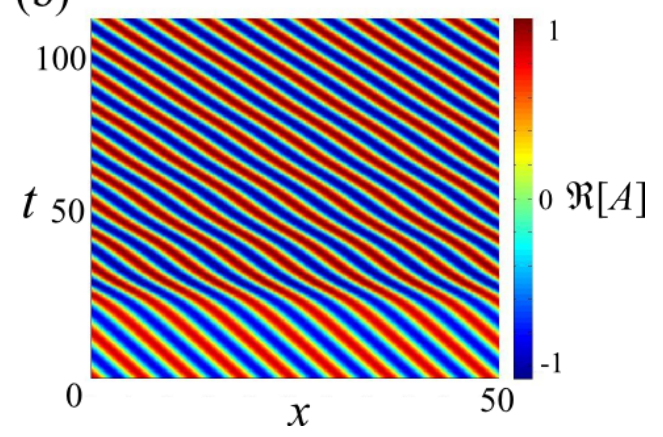

Fig. 20: Evolution of plane waves with $q=1$. The profile of $\Re[A]$ is shown in color. System parameters: $\tau=20, \eta=0.2, \beta=0.5, \epsilon=1, \mu=-1$, and $\nu=-0.1$. Panel (a) corresponds to the transition (a) in Fig. 19 when a defect is developed and the spatial wavenumber is changed. Panel (b) corresponds to the wavenumberpreserving transition (b) in Fig. 19 without defects. 

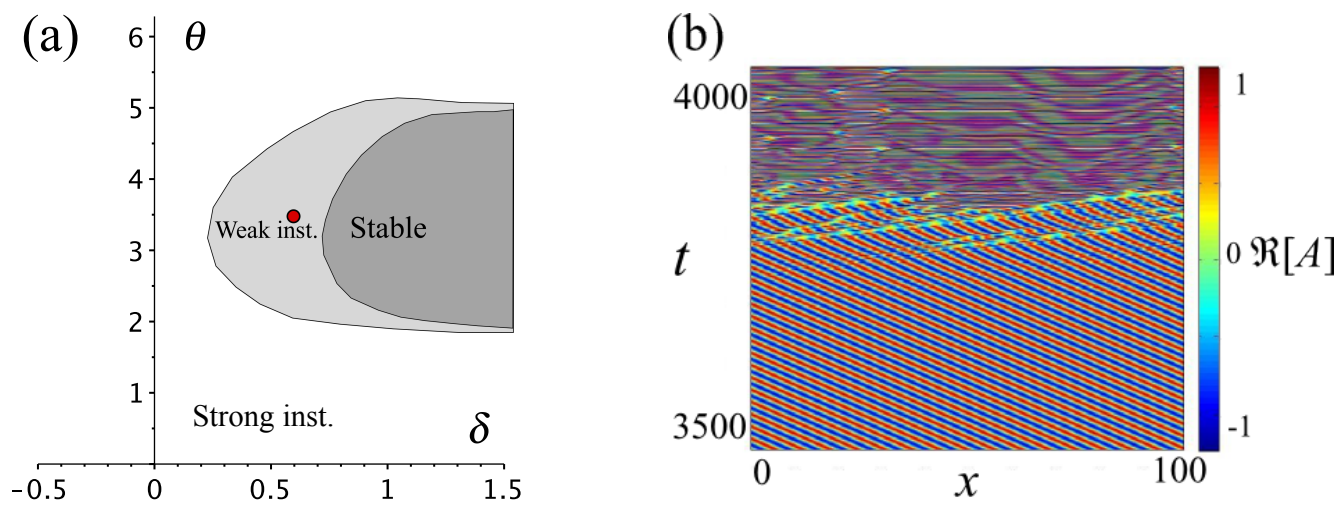

Fig. 21: A solution started from the vicinity of a weakly unstable plane wave with $q=1$ for large delay. The weak instability of the plane wave implies that the perturbation stays small at least several delay intervals. Initial unstable solution: $\theta=3.94, \omega=$ $0.33, a_{0}=1.05$ (red circle in (a)). System parameters: $\delta=0.56, \tau=50, \eta=0.2$, $\beta=0.5, \epsilon=1, \mu=-1$, and $\nu=-0.1$.

behavior, where the frequency of the snaking oscillations is proportional to the time delay $\tau$. Furthermore, stability properties of trivial homogeneous zero solution and plane wave solutions with different wavenumbers are investigated. The numerical bifurcation diagrams for various delay times as well as the analytical results in the limit of large delay reveal the borders of strong and weak instability of the plane waves. Direct numerical integration of the model equation confirms the results of analytical investigations.

\section{References}

[1] V. V. Afanasjev, N. N. Akhmediev, and J. M. Soto-Crespo. Three forms of localized solutions of the quintic complex Ginzburg-Landau equation. Physical Review E, 53(2):1931 - 1939, 1996.

[2] N. Akhmediev, A. Ankiewicz, and J. M. Soto-Crespo. Stable soliton pairs in optical transmission lines and fiber lasers. J. Opt. Soc. America B, 15:515-523, 1998.

[3] I. S. Aranson and L. Kramer. The world of the complex Ginzburg-Landau equation. Rev. Mod. Phys., 74(1):99-143, Feb. 2002.

[4] M. Cross and H. Greenside. Pattern formation and Dynamics in nonequilibrium systems. Cambridge Univ. Press, 2009.

[5] M. C. Cross and P. C. Hohenberg. Pattern formation outside of equilibrium. Rev. Mod. Phys., 65:851-1112, Jul. 1993.

[6] B. Fiedler, S. Yanchuk, V. Flunkert, P. Hövel, H.-J. Wünsche, and E. Schöll. Delay stabilization of rotating waves near fold bifurcation and application to all-optical control of a semiconductor laser. Phys. Rev. E, 77:066207, 2008.

[7] G. Giacomelli, F. Marino, M. A. Zaks, and S. Yanchuk. Coarsening in a bistable system with long-delayed feedback. EPL (Europhysics Letters), 99:58005, 2012.

[8] G. Giacomelli and A. Politi. Relationship between delayed and spatially extended dynamical systems. Phys. Rev. Lett., 76(15):2686-2689, 1996. 
[9] Ph. Grelu and N. Akhmediev. Group interactions of dissipative solitons in a laser cavity: the case of 2+1. Opt. Express, 12:3184-3189, 2004.

[10] S. V. Gurevich and R. Friedrich. Instabilities of localized structures in dissipative systems with delayed feedback. Phys. Rev. Lett., 110:014101, 2013.

[11] T. Heil, I. Fischer, W. Elsäßer, and A. Gavrielides. Dynamics of semiconductor lasers subject to delayed optical feedback: The short cavity regime. Phys. Rev. Lett., 87:243901, 2001.

[12] Y. S. Kivshar and G. Agrawal. Optical Solitons: From Fibers to Photonic Crystals. Elsevier Science, 2003.

[13] L. Larger, B. Penkovsky, and Yu. Maistrenko. Virtual chimera states for delayedfeedback systems. Phys. Rev. Lett., 111:054103, Aug. 2013.

[14] M. Lichtner, M. Wolfrum, and S. Yanchuk. The spectrum of delay differential equations with large delay. SIAM J. Math. Anal., 43:788-802, 2011.

[15] M. Matsumoto, H. Ikeda, T. Uda, and A. Hasegawa. Stable soliton transmission in the system with nonlinear gain. IEEE/OSA Journal of Lightwave Technology, 13:658-665, 1995.

[16] A. Mielke. The Ginzburg-Landau equation in its role as a modulation equation. In Handbook of dynamical systems, Vol. 2, pages 759-834. North-Holland, Amsterdam, 2002.

[17] K. A. Montgomery and M. Silber. Feedback control of traveling wave solutions of the complex Ginzburg-Landau equation. Nonlinearity, 17:2225-2248, 2004.

[18] J. Mørk, B. Tromborg, and J. Mark. Chaos in semiconductor lasers with optical feedback: theory and experiment. IEEE J. Quantum Electron., 28:93-108, 1992.

[19] A. C. Newell. Nonlinear Wave Motion, chapter Envelope Equations, pages 157-163. American Mathematical Society, Providence, Rhode Island, 1974.

[20] C. M. Postlethwaite and M. Silber. Spatial and temporal feedback control of traveling wave solutions of the two-dimensional complex Ginzburg-Landau equation. Physica D: Nonlinear Phenomena, 236(1):65-74, Dec. 2007.

[21] D. V. Ramana Reddy, A. Sen, and G. L. Johnston. Dynamics of a limit cycle oscillator under time delayed linear and nonlinear feedbacks. Physica D: Nonlinear Phenomena, 144:335-357, 2000.

[22] V. Rottschäfer and B. Krauskopf. The ECM-backbone of the Lang-Kobayashi equations: A geometric picture. Internat. J. Bifur. Chaos, 17:1575-1588, 2007.

[23] Wim van Saarloos and P. C. Hohenberg. Fronts, pulses, sources and sinks in generalized complex Ginzburg-Landau equations. Physica D, 56:303-367, 1992.

[24] J. Sieber, M. Wolfrum, M. Lichtner, and S. Yanchuk. On the stability of periodic orbits in delay equations with large delay. Discrete Contin. Dyn. Syst. A, 33:31093134, 2013. 
[25] M. C. Soriano, J. García-Ojalvo, C. R. Mirasso, and I. Fischer. Complex photonics: Dynamics and applications of delay-coupled semiconductors lasers. Rev. Mod. Phys., 85:421-470, Mar. 2013.

[26] J. M. Soto-Crespo, N. Akhmediev, and V. V. Afanasjev. Stability of the pulselike solutions of the quintic complex ginzburg-landau equation. J. Opt. Soc. Am. B., 13:1439-1449, 1996.

[27] J. M. Soto-Crespo and L. Pesquera. Analytical approximation of the soliton solutions of the quintic complex Ginzburg-Landau equation. Phys. Rev. E, 56(6):7288-7293, 1996.

[28] K. Staliunas, R. Herrero, and G. J. de Valcárcel. Arresting soliton collapse in twodimensional nonlinear schrödinger systems via spatio-temporal modulation of the external potential. Phys. Rev. A, 75:011604(R), 2007.

[29] O. Thual and S. Fauve. Localized structures generated by subcritical instabilities. J. Phys. (France), 49:1829-1833, 1988.

[30] M. Tlidi, A.G. Vladimirov, D. Pieroux, and D. Turaev. Spontaneous motion of cavity solitons induced by a delayed feedback. Phys. Rev. Lett., 103:103904, 2009.

[31] A. G. Vladimirov, S. V. Fedorov, N. A. Kaliteevskii, G. V. Khodova, and N. N. Rosanov. Numerical investigation of laser localized structures. Journal of Optics B: Quantum \& Semiclassical Optics, 1:101-106, 1999.

[32] M. Wolfrum, S. Yanchuk, P. Hövel, and E. Schöll. Complex dynamics in delaydifferential equations with large delay. Eur. Phys. J. Special Topics, 191:91-103, 2010 .

[33] S. Yanchuk and P. Perlikowski. Delay and periodicity. Phys. Rev. E, 79:046221, Apr. 2009.

[34] S. Yanchuk and M. Wolfrum. A multiple time scale approach to the stability of external cavity modes in the Lang-Kobayashi system using the limit of large delay. SIAM J. Appl. Dyn. Syst., 9:519-535, 2010. 